\title{
Use of Survivorship Care Plans to Re-Engineer Breast Cancer Follow-Up
}

\author{
Grace Makari-Judson*, Sandra Hubbard and Tamara Wrenn
}

\author{
Baystate Medical Center, Tufts University School of Medicine, Springfield, MA, USA
}

\begin{abstract}
Background: Optimal strategies for quality care of breast cancer survivors include providing value in each visit and appropriately utilizing resources. The purpose of this study was to demonstrate the use of Survivorship Care Plans (SCPs) to coordinate follow-up in a multidisciplinary practice and improve access to breast surgeons.

Methods: In 2009, our breast surgeons, medical oncologists and nurse practitioners agreed upon guidelines for follow up of breast cancer patients, developed a Survivorship Care Program to follow active treatment and implemented use of SCPs. To improve access to two part-time breast surgeons, guidelines were established to shift follow-up to medical oncologists and nurse practitioners. After diagnosis, patients were given comprehensive SCPs which included recommended follow up visits and testing. Wait times and numbers of new surgical patients were measured before and after use of SCPs.

Results: Wait times were measured from call to first appointment. New patients included both benign and malignant breast disease. Implementation of SCPs occurred during 2009. In 2007 and 2008, average wait times were 43.5 days and 28.5 days respectively. Following implementation of SCPs, wait times in 2010 and 2011 were 10 and 9 days respectively. Numbers of new patients seen were 573 and 486 in 2007 and 2008; 571 and 650 new patients were seen in 2010 and 2011.

Conclusions: SCPs were useful in re-engineering follow-up habits of clinicians, adding value to each visit and gaining acceptance from established patients regarding recommended surveillance. SCPs contributed to reduced wait times and increase in volume of new patients seen by breast surgeons. Future studies should assess contribution of SCPs to reducing unnecessary tests and improving compliance with ASCO guidelines.
\end{abstract}

Keywords: Survivorship care plans, breast cancer, follow-up.

\section{INTRODUCTION}

Most women diagnosed with breast cancer today have an $80-90 \%$ chance of being alive in ten years with most recent numbers showing continued improvement in survival of $30 \%$ since 1991 [1]. As breast cancer survivors continue to increase in number, their care has the potential to serve as a model for survivorship care in other adult cancers.

The call for survivorship research and the development of survivorship care plans (SCPs) was recommended by the Institute of Medicine in 2005 [2] with the goal of meeting the needs of a growing population. However, recent estimates still describe the use of SCPs as "a work in progress" [3]. SCP s are labor intensive and have been the focus of recent debate. Use of SCPs at the time of discharge from the oncologist's practice to the primary care provider was not associated with improved patient reported outcomes in one randomized trial [4]. However, benefits of SCPs may include enhanced compliance with guidelines and subsequent improvement in value in cancer care [5]. Unfortunately, data from the Quality Oncology Practice Initiative (QOPI) shows poor compliance with distribution of SCPs [6].

*Address correspondence to this author at the Baystate Medical Center, 376 Birnie Avenue, Springfield, MA 01199-0001, USA; Tel: 413-794-5433; Fax: 413-794-3613; E-mail: grace.makari-judson@baystatehealth.org
Although SCPs were intended to help patients understand disease and long term care goals, we saw this as an opportunity to communicate to the patient as well as other specialists involved in care, guideline based follow-up. The recommendation for clinical breast exam every six months interpreted by surgeon, medical oncologist and often radiation oncologists may translate to excess visits. Specifically, we faced a challenge of getting new breast cancer patients access to breast surgeon whose schedules were filled with follow-up visits. This shift enabled us to reduce follow up visits to surgeons, thus allowing for more new patients to be seen and for the wait times for new patients to drop precipitously.

Communication with breast cancer survivors about guideline based post-treatment follow-up may be improved with SCPs, however few studies have addressed measurable outcomes. We demonstrate how the survivorship care plan (SCP) and survivorship program (SP) were developed, accepted by the breast cancer specialists in our group and welcomed by our patients.

\section{METHODS}

Baystate Regional Cancer Program, includes the flagship program at Baystate Medical Center, Springfield Massachusetts, where a multidisciplinary team of clinicians focuses on breast cancer care. The Tumor Registry identifies 300-350 new breast cancer diagnoses per year. Prior to 2009, 
follow up was partly determined at physician discretion and partly patient-driven, often with both medical and surgical providers seeing patients within short spans of time. In 2009, our breast surgeons, medical oncologists, nurse practitioners (NP) and radiation oncologists agreed upon practice guidelines to coordinate follow-up for patients with early stage breast cancer. Our program included two part time breast surgeons, a surgical oncologist who covered as needed and two surgical NPs. Breast Medical Oncology included three medical oncologists and one nurse practitioner. Number of new breast cancer patients seen was provided by Tumor Registry.

\section{Consensus on Follow-Up}

Existing guidelines for follow-up care from the American Society of Clinical Oncology (ASCO) [7] and the National Comprehensive Cancer Network [8] were reviewed and our own guideline developed. Breast Surgery would see patients post-operatively, after completing radiation to assess cosmesis, and then only as needed. The medical oncologists would follow patients during systemic chemotherapy, then once every 3-6 months during years 1-2, and once every 6 months during years 2-5. After five years, patients would "graduate" to the newly established Survivorship Program. Patients on extended duration hormone treatment may still follow-up with the medical oncologist. Radiation Oncology followed patients for one to two years with different goals of follow-up. Providers were encouraged to use the phrase "I am still your doctor, if you need anything in the future, I am here for you" when describing coordination of care.

\section{Development of SCP}

The SCP (APPENDIX A) was developed following ASCO guidelines [7] and outlined for both patient and provider the recommended coordination of follow up care. SCP was posted at the workstations of all breast providers as a reminder to the agreed upon guidelines. SCP included information on diagnosis, stage and treatment, however, was not intended to be a treatment summary for patients receiving chemotherapy. All the providers are listed in the side panel by specialty, the SCPs describe symptoms that should be reported such as back or bone pain.

SCPs outlined the needed testing including mammography and consideration of genetic testing for those who qualified. Importantly, the SCP listed specific tests that are not required as supported by ASCO guidelines.

Distribution to breast cancer patients in the first 6 months of care was recommended usually at a follow-up visit by the medical oncologist. Chemotherapy treatment summaries were distributed separately. Surgeons had copies available to refer to and to be able to introduce the goals to the patients at the post operative visit. Distribution of SCPs occurred regularly during the first visit with the NP in the SP.

\section{Creation of Survivorship Program (SP)}

The Medical Oncology NP led the development of the Survivorship Program. The goals of the SP were to provide proper follow-up to detect local or distant relapse or second cancers, to manage health issues that may be treatment related and to promote wellness. Patients received a notebook which included educational information on topics such as heart health, osteoporosis, and management of menopausal symptoms (APPENDIX B). The annual visit included a physical exam, updated family history and mammogram performed the same day if possible.

In addition, a Wellness Program was available which consisted of quarterly evening educational workshops on topics such as exercise, healthful cooking and relaxation techniques. Each workshop included a health topic, leisure activity and fellowship. Our previously developed Annual Survivor's Day was expanded to include additional topics around wellness.

\section{Wait Times}

We measured wait time from patient/ referring provider call to first available appointment in calendar days. New patients included patients with either a diagnosis of breast cancer or undiagnosed lumps, or other breast problem. Wait time data does not include new breast cancer patients who were force booked by the triage nurse. Data is presented for the 2007 and 2008 (two years before implementation of SCPs) and 2010 and 2011 (two years after implementation of $\mathrm{SCP})$ for the two breast surgeons.

\section{RESULTS}

The recommendations for coordinating follow-up care were accepted by providers and patients. Discontinuation of follow-up visits by the breast surgery team was practice changing. Development of the nurse practitioner led SP eased the burden of follow up from the medical oncologist. Prior to 2009, although we had agreed in principle on coordination and frequency of follow up care, the medical oncologist and the breast surgeon would often see a given patient within a short span of time. Although we had previously addressed guidelines for follow-up care, implementation was challenging as it proved difficult to change behaviors and gain acceptance of practice guidelines even after endorsement.

Once the new guideline was put in place, patients who were already booked for follow-up appointments were not cancelled, but were given SCPs at the subsequent visit to explain why the surgeon would no longer be following them. The SCP helped ease the transition to a new work process for both patient and provider.

Implementation of SCPs occurred during 2009, which was a transition year. Data from the two years prior and the two years after is listed in Table $\mathbf{1}$. Wait times had started to decrease between 2007 and 2008 but were still high. In reality, new breast cancer patients were not waiting as long as the data suggests since they were scheduled using the less desirable "back door" approach. This form of scheduling is a burden on staff and may promote a perception of poor access to breast surgeons that may have impacted referrals and volumes in 2008. After 2009, wait times dramatically decreased as did the need for triage nurse to force book new patients.

Shorter wait times directly impacted on the number of new patients seen, allowing for an increase in total volume of new patients and the number of breast surgeries performed. Table 1 demonstrates a $46 \%$ increase in the number of breast surgeries performed between 2008 and 
2011. The number of new breast cancer patients seen by the breast surgery group and medical oncologists is presented in Table 2 where a clear increase in the number of new breast cancer patients occurred between 2007 and 2011. When fewer new breast cancer patients were seen by the breast surgeons, fewer new patients were seen by the medical oncologists since the surgeons generally direct the referrals. The parallel increase in new breast cancer patients seen by the medical oncologist indicates that there was no negative impact on those practices as a result of the shift in follow-up outlined by the SCPs. Other factors may have impacted on wait times such as the additional surgeon participation, however, with the dramatic reduction in wait times came a $34 \%$ increase in new breast cancer patients between 2007 and 2011 to the practices.

Table 1. The Change in Wait Time for a New Patient Visit to a Breast Surgery Group, Measured in Calendar Days, the Number of New Patients Seen, and the Number of Breast Surgeries Performed, Before and After the Implementation of SCPs

\begin{tabular}{|c|c|c|c|c|}
\hline & $\mathbf{2 0 0 7}$ & $\mathbf{2 0 0 8}$ & $\mathbf{2 0 1 0}$ & $\mathbf{2 0 1 1}$ \\
\hline \hline Wait time (days) & 43.5 & 28.5 & 10 & 9 \\
\hline \# New patients & 573 & 486 & 571 & 650 \\
\hline \# Breast surgeries performed & 159 & 184 & 186 & 397 \\
\hline
\end{tabular}

Table 2. Change in Numbers of New Breast Cancer Patients Seen in a Multidisciplinary Practice from 2007 and 2008 Prior to Implementation of SCPs to 2010 and 2011 Following Implementation of SCPs

\begin{tabular}{|c|c|c|c|c|}
\hline & $\mathbf{2 0 0 7}$ & $\mathbf{2 0 0 8}$ & $\mathbf{2 0 1 0}$ & $\mathbf{2 0 1 1}$ \\
\hline \hline $\begin{array}{c}\text { \# New Breast Cancer } \\
\text { Patients seen by Surgeons }\end{array}$ & 162 & 188 & 189 & 248 \\
\hline $\begin{array}{c}\text { \# New Breast Cancer Patients } \\
\text { seen by Medical Oncologists }\end{array}$ & 200 & 233 & 217 & 269 \\
\hline $\begin{array}{c}\text { \# New Breast Cancer Patients shared by } \\
\text { both Surgeons and Medical Oncologists }\end{array}$ & 184 & 227 & 218 & 247 \\
\hline
\end{tabular}

Patients being seen in the Survivorship Program were also invited to attend quarterly evening programs and an Annual Breast Cancer Survivor's Day. Participation in the Annual Survivorship Day increased from 270 in 2010, 320 in 2011 and 325 in 2012. The quarterly evening programs were attended by on average 30-50 participants. 117 women attended the first three evening workshops in 2010 [9]. Evaluation from two of these workshops is detailed in Table 3.

Table 3. Evaluation of Evening Wellness Programs

\begin{tabular}{|c|c|c|c|}
\hline $\begin{array}{c}\text { Evening } \\
\text { Program }\end{array}$ & \# Attendees & \# Respondents & $\begin{array}{c}\text { Did you Find the } \\
\text { Program Helpful? } \\
\text { \% Yes }\end{array}$ \\
\hline \hline Winter 2010 & 30 & 28 & 93 \\
\hline Spring 2010 & 32 & 32 & 82 \\
\hline
\end{tabular}

Note, spouses of breast cancer Survivors may have been in attendance but did not complete evaluation forms.

\section{DISCUSSION}

SCPs were useful in re-engineering follow-up habits of clinicians and facilitating coordinated care. Each provider worked "at the top of their license" allowing for a shift in the appointment types in our cancer program. The NP leading our new SP became responsible for follow-up visits of patients beyond five years, previously done by medical oncologists. Medical oncologists continued to perform the majority of the follow-up for the first five years, and took on primary responsibility for ordering follow-up imaging. The breast surgeons focused their practice on new patients and early post operative follow- up. Although we do not have data on the total number of visits per patient during the surveillance period, this was likely significantly decreased since surgeons tended to follow the patients every 6 months for five years. Acceptance of the SCP eliminated unnecessary visits, contributed to reduced wait times and increase in volume of new patients seen by surgeons. Because the surgeons generally direct the referrals to the medical oncologists, the numbers of new breast cancer patients seen by medical oncologists in the group also increased.

What makes good follow-up care and what is excessive? McCabe et al. describe a "misalignment of care whereby revenue-generating services (e.g. surveillance testing) may be overused and non- revenue generating services (e.g. aspects that might improve coordination of care) underused" [6]. As patients witness rising co-pays, making each visit count is appreciated. Cancer care in the future, like in medicine in general, needs to be anchored in value, defined by Porter [10] as health outcome achieved per dollar spent. Providing value in follow up care is of particular importance in preparing for the world of Accountable Care Organizations and global payments.

Breast cancer follow up guidelines are well known to the oncology community, yet surveillance patterns vary widely Margenthaler JA, et al. [11]. We were fortunate to get buy in on the SCP from the surgeons in our program. Ganz et al. [12] described excess visits even beyond three years, with patterns associated with the types of treatment. The frequency of follow up visits exceeded that recommended by ASCO and SCPs inconsistently provided to patients. We did not measure the frequency by which SCPs were given, however, even when not consistently given to the patient, the template was posted by the workstation of all of the providers as a reminder of the agreed upon follow up schedule. A previous effort to coordinate follow-up visits without SCPs resulted in poor compliance despite group consensus.

Who gives out the SCP and when? Presentation of the SCP by the medical oncology NP was a natural part of the patient's first visit to the SP. However, much of the information is valuable to patients after completing initial treatment when questions regarding long term follow up start to arise. Medical oncologists were encouraged to give the SCP in first six months after completion of chemotherapy and/or radiation, however many found it difficult to work into the time constraints of a typical visit.

It was anticipated that by eliminating routine follow up care by breast surgery, that capacity would increase for new 
patients. Time from diagnosis to visit with surgeon was one of the measures tracked by our breast health network overseeing quality. This metric, defined as less than five business days, was difficult to achieve, although a broader metric such as time from diagnosis to surgery less than 30 days was achievable.

A proposed benefit of SCPs is their use in communicating recommended testing to cancer survivors. A survey of 300 breast cancer survivors demonstrated overestimation of risk of recurrence and second cancers, raising concerns about patient requests for unnecessary testing [13]. Although our study did not track test utilization, the SCPs were helpful in documenting tests that were not recommended, thus facilitating exam room discussions. ASCO's Cost of Cancer Care Task Force cited use of unnecessary imaging and tumor markers in breast cancer follow up amongst the "top five" list to improve quality [14]. Studies to date have suggested ongoing concerns about excess tests for surveillance, even in patients receiving SCPs presenting ongoing opportunity to increase compliance [15].

Our standard for breast cancer follow up is applicable to other practices, both academic and community based, provided that there is acceptance on the frequency of follow up and the goals of follow up care. The medical oncologist provides follow up in the first five years or while patients are still on therapy. After five years, rather than having the medical oncologist continue follow up or transfer patients back to primary care providers, we established an NP driven SP. These annual visits are coordinated to take place at the same time as the annual mammogram and focus on healthy lifestyles, signs of recurrence and needs of survivors. Patients expressed that the uncertainty experienced at the end of active treatment is mitigated by remaining in a safe clinical environment that can easily be transitioned back to other diagnostic and clinical services. Patients expressed satisfaction in having a SCP and knowing the expectations.

\section{CONCLUSION}

By prompting providers and reassuring patients, SCPs were useful in coordinating care, reducing unnecessary visits thereby improving access for new patients. Future studies should assess contributions of SCPs to reducing unnecessary tests and improving compliance with published guidelines.

\section{CONFLICT OF INTEREST}

The authors confirm that there is no conflict of interest.

\section{ACKNOWLEDGEMENTS}

Rays of Hope - A Walk Towards a Cure for Breast Cancer, Springfield Ma for support for education materials and programs for breast cancer survivors.

Members of the Baystate Health Breast Network for support of guideline based care.

\section{APPENDIX A}

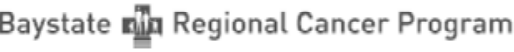

BAYSTATE HEALTH BREAST NETWORK
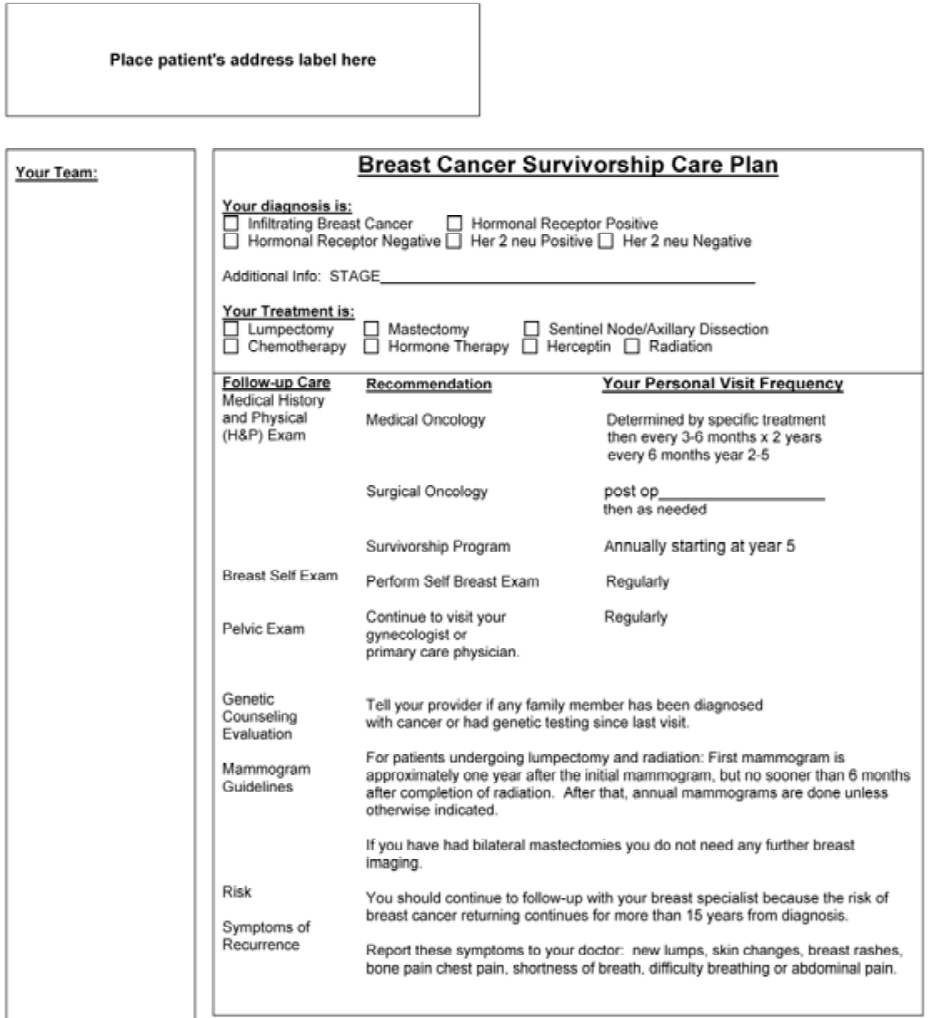

Your team will make every effort to coordinate yearly vists with mammograms 


\section{APPENDIX B}

\section{BREAST CANCER SURVIVORSHIP PROGRAM}

\section{Table of Contents}

1. Breast Cancer Survivorship Care Plan

2. Introduction

3. Algorithm for Taking Care of Your Health

4. Alcohol Use and Breast Cancer

5. Breast Cancer Patient Resource List

6. Exercise and Healthy Weight Maintenance

7. Exercise Resource Guide

8. Genetic Testing/Counseling

9. Heart Health and Cholesterol

10. Hot Flashes

11. Joint Pain

12. Lymphedema

13. Mediterranean Diet

14. Prevention of Osteoporosis, Bone Health

15. Senior Center Resource Guide

16. Sexual Health

17. Smoking and Breast Cancer

18. Stress, Depression and Anxiety

19. Tips For A Good Night's Sleep

20. Vaginal Dryness

21. Vitamin D

22. Water, Drink to Your Health
23. Weight-Bearing Exercises \& Bone Health in Breast Cancer Survivors

\section{REFERENCES}

[1] Siegel R, Naishadham D, Jemal A. Cancer statistics, 2013. CA Cancer J Clin 2013; 63: 11-30.

[2] Institute of Medicine Committee on Cancer Survivorship: From Cancer Patient to Cancer Survivor: Lost in Translation, Hewitt, Ed. Washington, DC, National Academies Press, 2005.

[3] Ganz PA, Earle CC, Goodwin PJ. Update on progress of cancer survivorship care and research. J Clin Oncol 2012; 30(30): 3655-6.

[4] Grunfeld E, Julian JA, Pond G, et al. Evaluating survivorship care plans: Results of randomized clinical trial of patients with breast cancer. J Clin Oncol 2011; 29: 4755-62.

[5] Smith TJ, Synder C. Is it time for (Survivorship care) Plan B? J Clin Oncol 2011; 29: 4740-2.

[6] McCabe MS, Bhatia S, Oeffinger KC, et al. Statement: Achieving high-quality cancer survivorship care. J Clin Oncol 2013; 46: 1-11.

[7] Khatcheressian JL, Wolff AC, Smith TJ, et al. Update of the breast cancer follow-up and management guidelines in the adjuvant setting. J Clin Oncol 2006; 24: 5091-7.

[8] Carlson RW, Allred DC, Anderson BO. Breast cancer practice guidelines. J Natl Compr Canc Netw 2009; 7: 122-92.

[9] Hubbard S, Carpenter-Gleason C, Tipton C, Mertens W, MakariJudson G. S.O.S- supporting our survivors, focus on wellness. National consortium of breast centers annual meeting March 2010 Am J Clin Oncol 2010.

[10] Porter M. What is value in health care? N Engl J Med 2010; 363 2477-81.

[11] Margenthaler JA, Allam, E Chen L, et al. Surveillance of patients with breast cancer after curative intent primary treatment: current practice patterns. J Oncol Pract 2012; 8(2): 79-83

[12] Ganz A. Quality of care amongst breast cancer survivors in the Univ of California Athena Breast Health Network. J Clin Oncol 2012; 30(suppl 34): 164.

[13] Makari-Judson G, Loke C, Katz D, et al. Knowledge of prior treatments and appreciation of future health concerns in breast cancer survivors. [Manuscript submitted 2013].

[14] Schnipper L, Smith T, Raghavan D, et al. American society of clinical oncology identifies five key opportunities to improve care and reduce costs: The top five list for oncology: J Clin Oncol 2012; 30: $1715-24$.

[15] Hahn E, Mody K, Jacobson A, et al. Use of advanced imaging and biomarker tests for post treatment surveillance in early stage breast cancer survivors. J Clin Oncol 2012; (Suppl 34): abstract 168. 